\title{
Choice of hybrid functional and basis set optimization to calculate the structural, electronic, mechanical, and vibrational properties of $\mathrm{BaSnO}_{3}$
}

\author{
Thiago M. Duarte ${ }^{1} \cdot$ Prescila G. C. Buzolin $^{1} \cdot$ Ieda M. G. Santos $^{2} \cdot$ Elson Longo $^{3}$ • \\ Julio R. Sambrano ${ }^{1}$
}

Received: 7 December 2015 / Accepted: 25 April 2016 / Published online: 20 May 2016

(C) Springer-Verlag Berlin Heidelberg 2016

\begin{abstract}
This work aimed to study the influence of seven hybrid functionals (B3LYP, B1WC, B3PW, PBE0, PBESOL0, SOGGAXC, and WC1LYP) and selected basis sets in the CRYSTAL software library on the calculated structural, electronic, mechanical, and vibrational properties of $\mathrm{BaSnO}_{3}$ under the framework of periodic density functional theory. Based on the results showing the best approximation of the experimental band gap energy and lattice parameter, the PBE0 functional and the Baranek basis set were used to optimize the outer valence shell functions, $\alpha_{\mathrm{sp}}$ and $\alpha_{\mathrm{d}}$, in order to improve the quality of calculated properties. An analysis was done to determine the density of states, band structure, elastic constants, and infrared (IR) active modes. The results were compared with other reported quantum mechanical calculations and available experimental data.
\end{abstract}

Keywords $\mathrm{BaSnO}_{3} \cdot$ Perovskite $\cdot \mathrm{CRYSTAL} \cdot$ Hybrid functional $\cdot$ Basis set

Published as part of the special collection of articles "CHITEL 2015 - Torino - Italy".

Electronic supplementary material The online version of this article (doi:10.1007/s00214-016-1901-1) contains supplementary material, which is available to authorized users.

Julio R. Sambrano

sambrano@fc.unesp.br

1 Grupo de Modelagem e Simulação Molecular, UNESP, Bauru, SP 17033-360, Brazil

2 Laboratório de Combustíveis e Materiais, UFPB, João Pessoa, PB 58051-900, Brazil

3 Instituto de Química, CDMF, UNESP, Araraquara, SP 14801-907, Brazil

\section{Introduction}

Alkaline earth stannates $\mathrm{ASnO}_{3}(\mathrm{~A}=\mathrm{Ca}, \mathrm{Sr}$, and $\mathrm{Ba})$ with the perovskite structure are important materials for a vast range of industrial applications, such as the electronics industry, not only due to the ease of modification of their chemical and physical properties [1], but also because of their high-temperature stability [2]. These stannates have been used to develop chemical sensors [3], stable capacitors [4], and water photoelectrolysis systems [5, 6].

There are many methods to synthesize perovskite, such as the solid-state reaction [7-10], hydrothermal [11-13], and modified sol-gel [14] methods, and the most popular in recent times, the microwave-assisted hydrothermal and solvothermal method [15, 16]. The last one has demonstrated efficiency in the processing of many compounds. Each method has its intrinsic complexity and can establish many important aspects and properties of materials.

Among these, $\mathrm{BaSnO}_{3}$ is an n-type semiconducting material which is normally reported to have a band gap energy of $3.1 \mathrm{eV}[17,18]$ and is stable at temperatures up to $1000{ }^{\circ} \mathrm{C}[2,19]$.

From an experimental point of view, according to Prodjosantoso et al. [20] who studied the system $\mathrm{Ba}_{1-x} \mathrm{SrSnO}_{3}$ obtained from the solid-state method of synthesis, the lattice parameter, $a$, of $\mathrm{BaSnO}_{3}$ is $4.1168 \AA$. This is the same value presented by Liu et al., $a=4.1163 \AA$ [18], and close to that presented by Smith et al., $a=4.117 \pm 0.002 \AA$ [21]. On the other hand, theoretical data obtained for the same cubic perovskite $\left(\mathrm{BaSnO}_{3}\right)$ using the generalized gradient approximation (GGA) and the local-density approximation (LDA) underestimated or overestimated the lattice parameter $a$, according to Moreira (4.142 $\AA$ (GGA); $4.045 \AA$ (LDA)) [22] and Bouhemadou (4.1916 $\AA$ (GGA); $4.059 \AA$ 
(LDA)) [23], as well as Wang (4.1236 $\AA$ ) [24] who performed a semiempirical study.

Moreira et al. [22] also simulated the electronic band structure, density of states (DOS), dielectric function, optical absorption, and infrared spectrum. The calculated indirect band gaps $(R \rightarrow \Gamma), 1.51 \mathrm{eV}(\mathrm{LDA})$ and $0.74 \mathrm{eV}$ (GGA), are much smaller than the experimental value.

Soleimanpour and Kanjouri [25] calculated the electronic, structural, and optical properties of cubic perovskite $\mathrm{BaSnO}_{3}$ using full-potential linearized augmented plane wave (FP-LAPW)-based density functional theory (DFT). Exchange and correlation effects are taken into account by using a GGA and an orbital independent modified BeckeJohnson potential coupled with GGA. The calculated indirect band gap $(R \rightarrow \Gamma)$ is $2.65 \mathrm{eV}$. This modification was the best approach to modeling the band gap. Furthermore, they have analyzed the inter-band contribution to the optical properties using the calculated band structure and density of states.

Bouhemadou and Haddadi [23] used first-principle calculations in order to investigate the structural, elastic, electronic, and thermal properties. Interactions of valence electrons with ion cores were represented by the Vanderbilt-type ultrasoft pseudopotential, and the exchange-correlation potential was used with the LDA, as well as the GGA. They found the indirect band gaps $(R \rightarrow \Gamma)$ to be $0.9722 \mathrm{eV}$ (LDA) and $0.070 \mathrm{eV}$ (GGA).

According to the two papers of Singh et al. [26, 27], the calculated band gap of $\mathrm{BaSnO}_{3}$ obtained by the FP-LAPW is 3.0 and $3.18 \mathrm{eV}$, respectively. Wang et al. [15] found the band gap value of $0.75 \mathrm{eV}$ for DFT method. Using HSE06 functional, implemented in the VASP code, Kim et al. [28] presented a theoretical band gap of $2.48 \mathrm{eV}$.

Observing the previously cited works, once more it is evident that an important step in the accuracy of DFT calculations is the choice of the functional. Also it is well known that some functionals are able to reasonably predict structures, but sometimes underestimate the band gap, such as GGA and LDA, and other functionals that describe the band gap well can often overestimate the lattice parameter. There are several comparative studies devoted to comparing the accuracy of the functional to predicting the structural and electronic properties of molecules and solids [29-32]. The main conclusion is that none of the available functionals are able to describe all the electronic, structural, mechanical, and vibrational properties of studied systems at the same time, and their results are strongly dependent on the basis set or the potential to be used.

In this sense, the aim of this research was to accurately study the influence of seven hybrid functional and several groups of available basis set (all electron and pseudopotential) upon theoretical calculations. To improve our knowledge, a systematic study addressing the performance of different basis sets and the possible necessity of a new optimization of these basis sets for accurately describing the properties of $\mathrm{BaSnO}_{3}$ is still necessary.

The simulations were done in order to improve the knowledge of the structural, electronic, mechanical, and vibrational properties related to the choice of functional and/or basis set, and they will be discussed in terms of the ability to reproduce the experimental data, with emphasis in the lattice parameter and band gap energy. An accurate calculation of the band gap is a fundamental step in simulations applied to solid-state chemistry.

A theoretical analysis was done to determine density of states (DOS), band structures, elastic constants, and infrared (IR) active modes. These results were compared with reported quantum mechanical calculations as well as available experimental data.

\section{Computational method and model system}

The simulations of $\mathrm{BaSnO}_{3}$ were done within the framework of periodic DFT using seven different hybrid functionals: B3LYP [33, 34], B1WC [35], B3PW [33, 3638], PBE0 [39], PBESOL0 [39], SOGGAXC [40], and WC1LYP [41] implemented in the CRYSTAL14 [42] software. This code uses a Gaussian-type basis set to represent crystalline orbitals as a linear combination of Bloch functions defined in terms of local functions (atomic orbitals). An overview of the algorithms used in the introduction of DFT in the CRYSTAL computer code is presented by Towler et al. [43]. An important step in the process of calculation is the choice of density functional approximations that has been successful for describing properties in solid state when they depend on accurate approximations of the band gap and band structure.

Barium, tin, and oxygen atoms were described by the SC_HAYWSC-31 $(1 d)$ G_baranek [44], ECP28MDF411(51d)G_baranek [45], and 8-411(1d)G baranek basis set [45], respectively. These basis sets were used for computational simulations of $\mathrm{BaZrO}_{3}, \mathrm{SnTiO}_{3}$, and $\mathrm{CsTaO}_{3}$ systems, respectively, and also are available in the CRYSTAL basis set library (http://www.crystal.unito.it/basis-sets. php).

These basis sets were chosen because they have been used for other families of perovskite; however, another available combination of basis sets for $\mathrm{Ba}, \mathrm{Sn}$, and $\mathrm{O}$ atoms was tested and evaluated previously.

In a general sense, the development of a basis set is not necessary from the "zero point," because many starting basis sets are already available on the CRYSTAL library. However, many of these basis sets are pre-optimized for other systems, so when we use different basis sets that have already been used for other systems, it may be necessary 

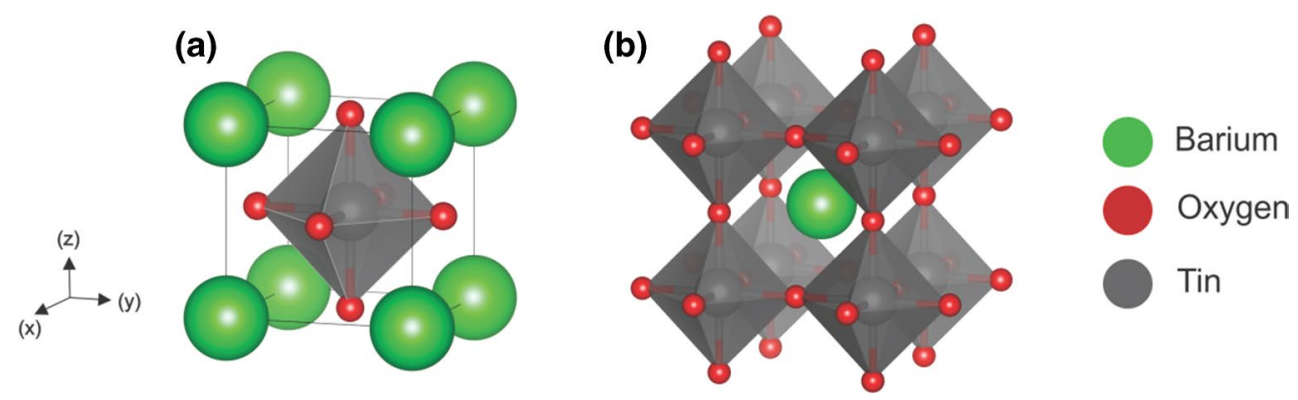

Fig. 1 Primitive unit cell of $\mathrm{BaSnO}_{3}$

to perform a new optimization in order to adapt a basis set to a new system. From this initial guess, all exponents and/ or contraction coefficients and the outer valence functions should be re-optimized.

On the other hand, a new optimization of the basis set is not always effective; it may happen that the optimization does not bring an improvement in the results.

In this sense, it will be demonstrated that this is an important step to before doing solid-state computational simulations.

What follows is a description of the optimization of the exponents for the outer valence shell functions $\alpha_{\mathrm{sp}}$ and $\alpha_{\mathrm{d}}$, in order to minimize the total energy of the structure at experimental parameters. Powell's algorithm method was used to perform the basis set optimization procedure to a convergence energy of $10^{-6}$ hartree.

The level of accuracy for the Coulomb and exchange series was controlled by a set of five thresholds $\left(10^{-7}\right.$, $10^{-7}, 10^{-7}, 10^{-7}$, and $\left.10^{-14}\right)$, and the shrinking factor (Pack-Monkhorst and Gilat net) was set to 6 corresponding to $100-\mathrm{k}$ points in the irreducible Brillouin zone.

The ideal cubic $\mathrm{BaSnO}_{3}$ structure belongs to the space group $\operatorname{Pm} \overline{3} \mathrm{~m}$, where the $\mathrm{Ba}$ atoms share the vertices of the unit cell and the Sn atoms are located at the body center, surrounded by six $\mathrm{O}$ atoms occupying the face centers in an octahedral configuration (Fig. 1a). Moreover, the cubic perovskite structure consists of a $\mathrm{Ba}$ atom coordinated with 12 oxygen atoms in a dodecahedral site and coordinated by eight octahedral $\mathrm{SnO}_{6}$ (Fig. 1b) [46].

\section{Results and discussion}

\subsection{Structural parameter and basis set optimization}

Figure $2 \mathrm{a}$ shows the calculated percentage error for the lattice constant values when minimizing the total energy of $\mathrm{BaSnO}_{3}$ with the original Baranek basis set for all tested hybrid functionals, and Table S1 (supplementary information) depicts the respective calculated values.
The results overestimate the experimental lattice parameter by $0.32-1.9 \%$ for all functionals, except to PBESOL0, which is $0.07 \%$ lower than the experimental lattice parameter. However, it was observed that all functionals underestimate the band gap energy by -11.29 to $-85.16 \%$, and in particular, PBE0 and PBESOL0 underestimate the band gap by -11.29 and $-21.61 \%$.

Based on which functional provided best approximation of the experimental band gap energy and lattice parameter, PBE0 and PBESOL0 (Fig. 1a) were used to optimize the outer valence shell functions, $\alpha_{\mathrm{sp}}$ and $\alpha_{\mathrm{d}}$, of the selected basis set.

Despite the PBESOL0 giving good results for the lattice parameters, but poor results for the band gap, we also performed the basis set optimization with this functional.

The results of these optimizations are shown in Table 1, and the complete description of these basis sets is available as supplementary information.

In addition, the basis set optimization was also performed with SOGGAXC functional. This functional showed very good result for the lattice parameter and thus can be compared to PBE0 and PBESOL0 functionals (see Fig. 2a). After the optimization, it was observed that the calculated lattice parameter was very close to the calculated value before optimization, and the band gap energy did not show any improvement.

Once more it is shown here that the basis set optimization may not be efficient if the accuracy of the desired calculated parameters or properties is already close to the experimental data.

The basis set optimization with PBESOL0 showed good agreement, keeping the lattice parameter, $a=4.11 \AA$, practically unchanged and given a considerable improvement in the band gap energy calculation with an underestimation of $\sim 4 \%(2.97 \mathrm{eV})$. On the other hand, the basis set optimization with PBE0 gave $a=4.145 \AA$ and a band gap energy of $3.13 \mathrm{eV}$. It is observed that after the basis set optimization with PBE0, the error in the calculated band gap is considerably improved, but the calculated structural parameter remains worse than for the optimization with PBESOLO. 
Fig. 2 a Calculated percentage error for the lattice parameters and band gap energy with the original Baranek basis set for all tested hybrid functionals. b Calculated percentage error for the lattice parameters and band gap with the optimized basis set with the PBESOL0 functional. The origin is set to the experimental lattice parameter, $a=4.1168 \AA$ [20], and band gap energy, $3.1 \mathrm{eV}$ [17]
Table 1 Optimized outer valence shell functions, $\alpha_{\mathrm{sp}}$ and $\alpha_{\mathrm{d}}$, of selected basis sets
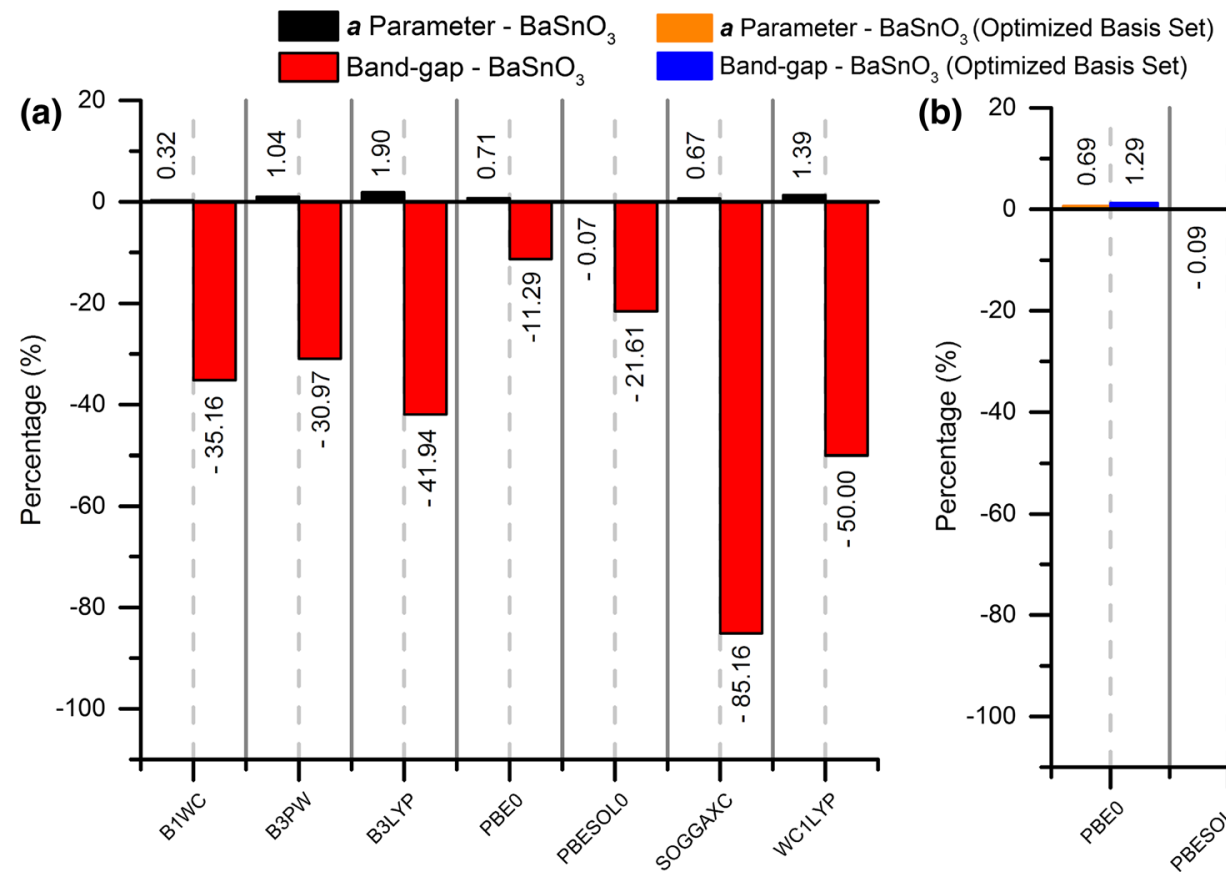

(b) 207

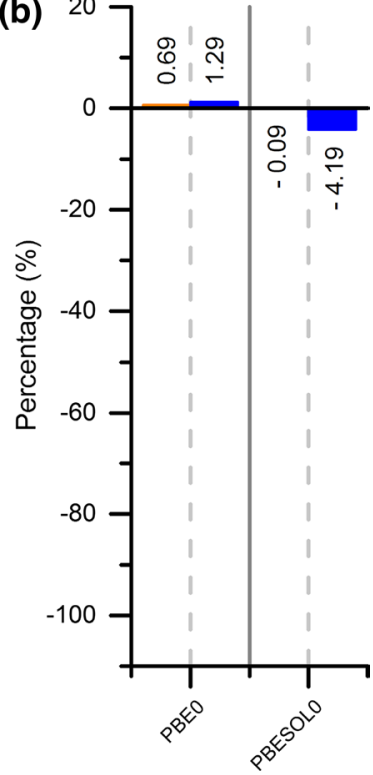

\begin{tabular}{lllllll}
\hline & $\mathrm{Ba}^{\mathrm{a}}$ & $\mathrm{Sn}^{\mathrm{a}}$ & $\mathrm{O}^{\mathrm{a}}$ & $\mathrm{Ba} \mathrm{[44]}$ & $\mathrm{Sn} \mathrm{[45]}$ & $\mathrm{O}$ [45] \\
\hline${ }^{1} \alpha_{\mathrm{sp}}$ & 0.2122485 & 0.9782922 & 0.4528085 & 0.217351 & 0.9800857 & 0.4526392 \\
${ }^{1} \alpha_{\mathrm{sp}}$ & - & 0.2199999 & 0.1485983 & - & 0.2036470 & 0.1505710 \\
${ }^{1} \alpha_{\mathrm{d}}$ & 0.3355518 & 0.3157988 & 0.6000000 & 0.323392 & 0.3201681 & 0.6316931 \\
${ }^{2} \alpha_{\mathrm{sp}}$ & 0.2114822 & 0.9800795 & 0.4529083 & - & - & - \\
${ }^{2} \alpha_{\mathrm{sp}}$ & - & 0.2199998 & 0.1482566 & - & - & - \\
${ }^{2} \alpha_{\mathrm{d}}$ & 0.3333274 & 0.3180610 & 0.6000768 & - & - & - \\
\hline
\end{tabular}

${ }^{\mathrm{a}}$ This work with ${ }^{1} \mathrm{PBE} 0$ and ${ }^{2} \mathrm{PBESOL} 0$
Therefore, based on the results showing the best approximation of the band gap energy and lattice parameter, the DOS, band structure, elastic constants, and IR spectrum analyses were calculated using the PBE0 functional.

\subsection{Electronic properties}

Figure 3 represents the band structure and the total and projected DOS of bulk $\mathrm{BaSnO}_{3}$. The top of the valence band (VB), coincident with the origin, is located at the $\mathrm{R}$ point, and the bottom of conduction band (CB) is located at the $\Gamma$ point; therefore, the calculated indirect band gap is $3.14 \mathrm{eV}$. This value is only $0.03 \mathrm{eV}$ greater than the experimental value. It is observed that the higher CB between the $\mathrm{M}$ and $R$ points is flat and the indirect gap between the $M$ and $\Gamma$ points is $2.75 \mathrm{eV}$, slightly higher the band gap between the $\mathrm{R}$ and $\Gamma$ points.

An analysis of the principal AO components of selected bands indicates that the upper VB is predominantly formed from oxygen $2 p_{\mathrm{x}} p_{\mathrm{y}}$ and $2 p_{\mathrm{z}}$ atomic orbitals, $5 p_{\mathrm{x}} p_{\mathrm{y}}$ and $5 p_{\mathrm{z}}$ atomic orbitals of $\mathrm{Ba}$ in the intermediate position, and $4 d_{x^{2}-y^{2}}$ and $4 d_{x y}$ atomic orbitals of Sn. These orbitals are separated from the CB by an indirect gap, which is composed of two different energy states. The first is due to the $\mathrm{O} 2 p_{\mathrm{x}} p_{\mathrm{y}}$ and $2 p_{\mathrm{z}}$ atomic orbitals, and the second is due to the $\mathrm{Ba} 4 d_{x^{2}-y^{2}}$ and $4 d_{x y}$ atomic orbitals.

The $\mathrm{Sn}-\mathrm{O}$ bond length in the tetrahedral structure is $2.07 \AA$, whereas the bond overlap population is $154 \mathrm{mlel}$. The $\mathrm{Ba}-\mathrm{O}$ bond length and bond overlap are $2.93 \AA$ and 25 mlel, respectively. The Mulliken charges were 1.84, 2.07 , and -1.31 lel, respectively, for $\mathrm{Ba}, \mathrm{Sn}$, and $\mathrm{O}$ atoms, respectively. These values show that the $\mathrm{Ba}-\mathrm{O}$ bond has greater ionic character, whereas the $\mathrm{Sn}-\mathrm{O}$ bond has greater covalent character $[6,47]$.

\subsection{Elastic constants}

Beyond the electronic and structural properties of a material, its mechanical properties can provide important information. Understanding the elastic properties of a material 
Fig. 3 a Band structure and $\mathbf{b}$ DOS of bulk $\mathrm{BaSnO}_{3}$

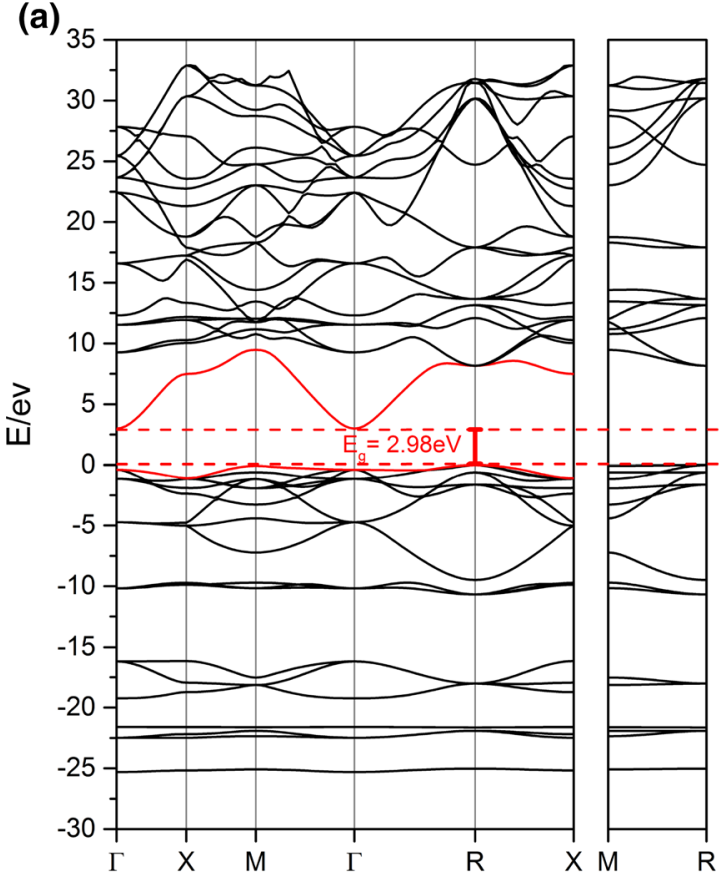

(b)

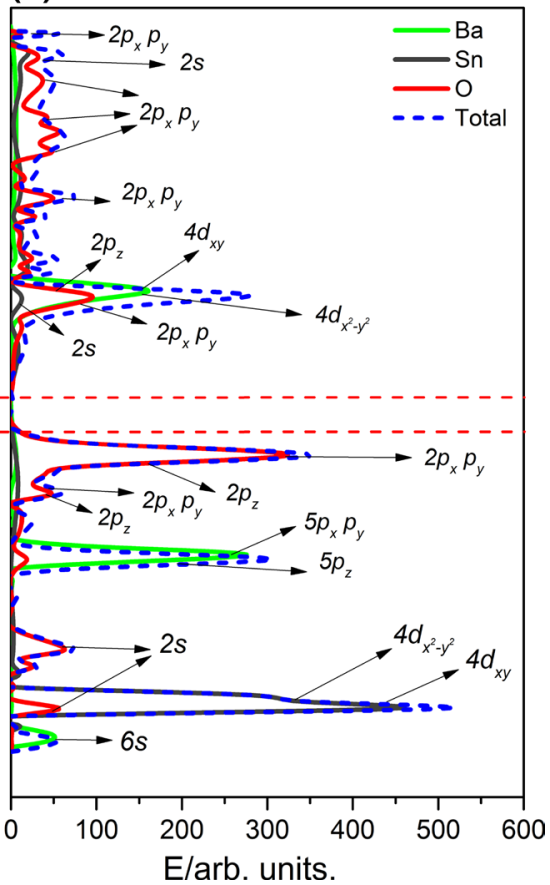

is crucial because they control the reversible response to external forces, determining a material's performance and its potential for possible applications. From the elastic constants, the bulk, shear, and Young's moduli can be determined. These properties are hard to quantify experimentally, especially for nanoscale materials.

Several theoretical studies have been devoted to elastic constants [30, 48, 49], and to the best of our knowledge, experimental measurements of elastic constants for $\mathrm{BaSnO}_{3}$ cubic phase are not available.

From the optimized cell parameters of $\mathrm{BaSnO}_{3}$, the elastic constants were calculated using a Taylor expansion in terms of the strain components and truncated to the second order.

There are only three constants to be determined for a cubic system, $C_{11}, C_{12}$, and $C_{44}$. The first constant expresses the distortion in the $x$ Cartesian direction. The second one represents the simultaneous deformation along the $x$ - and $y$-axes, while keeping constant in the $z$ Cartesian direction. The last one represents a simultaneous deformation in planes $x O z$ and $y O z$ and can be interpreted as a constant shear. The bulk modulus, $B_{0}$, for a cubic system can be calculated using $B_{0}=\left(C_{11}+2 C_{12}\right) / 3$.

There is not one unique method to determine the bulk modulus, and another way to estimate it is via several used formulations of the equation of state [50-54]. Therefore, our applied formula to estimate the bulk modulus is an alternative and simpler way to calculate $B_{0}$ and can be compared with other computational simulation strategies.

Table 2 shows the calculated PBE0 and PBESOLO values and other reported theoretical results. An evaluation of the
Table 2 Theoretical elastic constants $C_{\mathrm{ij}}(\mathrm{GPa})$ and bulk modulus $B_{0}$ in $\mathrm{GPa}$

\begin{tabular}{lllll}
\hline & \multicolumn{2}{l}{ Elastic constants } & \multirow{2}{*}{$B_{0}^{\mathrm{b}}$} \\
\cline { 2 - 4 } & $C_{11}$ & $C_{12}$ & $C_{44}$ & \\
\hline PBE0 $^{\mathrm{a}}$ & 296.33 & 89.13 & 111.07 & 158.20 \\
PBESOL0 $^{\mathrm{a}}$ & 319.53 & 95.09 & 114.86 & 169.91 \\
GGA [22] & 251.0 & 82.3 & 98.1 & 139.0 \\
LDA [23] & 358.5 & 93.5 & 110.3 & 182.0 \\
GGA [23] & 285.2 & 68.5 & 84.3 & 140.7 \\
GGA [25] & - & - & - & 146 \\
Exp [4]. & - & - & - & 145.8 \\
\hline
\end{tabular}

${ }^{\text {a }}$ Results obtained in the present work with basis set optimization

b Calculated from $B_{0}=\left(C_{11}+2 C_{12}\right) / 3$

seven hybrid functional results shows that $C_{11}, C_{12}$, and $C_{44}$ range from 261.76 to $319.53,80.39$ to 95.09 , and 101.13 to $114.86 \mathrm{GPa}$, respectively. The results for other functionals are shown in Table S2 (supplementary information).

PBE0 shows better agreement with the reported GGA results [22, 23, 25] than with the reported LDA results [23] and underestimates the PBESOL0 data. Nevertheless, PBESOL0 shows a reasonable agreement with the LDA results [23]. On the other hand, the overall GGA values also show a reasonable agreement with each other and are underestimated relative to the LDA and PBESOLO results. The same behavior is observed for the $B_{0}$ values. The differences between the calculated values are due to the choice of functional and quantum description of atoms. 
Table 3 Active infrared (IR) frequencies for all hybrid functionals and for PBESOL0 with optimized basis set

\begin{tabular}{lllll}
\hline IR & \multicolumn{2}{l}{$\mathrm{F}_{1 \mathrm{u}}\left(\mathrm{cm}^{-1}\right)$} & & $\mathrm{F}_{2 \mathrm{u}}\left(\mathrm{cm}^{-1}\right)^{\mathrm{c}}$ \\
\hline PBE0 $^{\mathrm{a}}$ & 145.81 & 255.11 & 632.80 & 242.81 \\
PBESOL0 $^{\mathrm{a}}$ & 150.38 & 245.99 & 657.96 & 259.33 \\
B3LYP $^{\mathrm{b}}$ & 131.81 & 241.84 & 591.51 & 226.98 \\
B1WC $^{\mathrm{b}}$ & 145.19 & 247.13 & 648.94 & 230.59 \\
B3PW $^{\mathrm{b}}$ & 140.27 & 248.28 & 625.87 & 233.37 \\
PBE0 $^{\mathrm{b}}$ & 146.01 & 257.81 & 632.24 & 244.06 \\
PBESOL0 $^{\mathrm{b}}$ & 150.19 & 247.71 & 658.59 & 261.48 \\
SOGGAXC $^{\mathrm{b}}$ & 137.93 & 215.30 & 625.76 & 197.80 \\
WC1LYP $^{\mathrm{b}}$ & 134.30 & 240.30 & 613.57 & 221.83 \\
HSE06 [28] & 131.3 & 253.6 & 620.6 & 237.5 \\
Exp. [9] & - & - & 640 & - \\
Exp. [14] & $\sim 150$ & $\sim 420$ & $\sim 735$ & - \\
Exp. [55] & - & - & 629 & - \\
Exp. [56] & - & - & 640 & - \\
Exp. [57] & - & - & 630 & - \\
Exp. [58] & - & - & 626 & - \\
Exp. [59] & - & - & 627 & - \\
Exp. (average) & 150 & 420 & 632 & - \\
\hline
\end{tabular}

${ }^{a}$ Results obtained in the present work with basis set optimization

b Results obtained in the present work

${ }^{c}$ Inactive mode

\subsection{Vibrational frequencies}

In a crystalline structure, the atoms can move relative to their equilibrium positions; therefore, the dynamics of these movements can provide knowledge about important chemical and physical properties.

The analysis of the vibrational modes and their corresponding frequencies is important to the prediction of structural stability, vibrational spectra, and thermodynamic properties. The vibrational frequencies were calculated at the $\Gamma$ point using the numerical second derivatives of the total energies.

The cubic structure has normal modes with the irreducible representation, $3 \mathrm{~F}_{1 \mathrm{u}}+\mathrm{F}_{2 \mathrm{u}}$ [55]. Three degenerate $\mathrm{F}_{1 \mathrm{u}}$ modes are active in the infrared (IR) and correspond to the acoustic phonon mode, and $F_{2 u}$ is inactive in the IR and corresponds to an optically silent mode.

Table 3 shows the calculated IR modes and other theoretical and experimental data, and Figs. 4 and 5 depict an infrared spectrum and the assignment of the dynamics in the crystalline structure. The results for other functionals are shown in Table S3 (supplementary information).

The most intense IR absorption peak occurs at $\sim 255 \mathrm{~cm}^{-1}$, representing the angle bending of $\mathrm{Sn}-\mathrm{O}-$ $\mathrm{Sn}$ along the $y$-axes with angular variation of $\mathrm{O}-\mathrm{Sn}-\mathrm{O}$ along the $z$-axes (Fig. $4 \mathrm{~b}$ ). The band at $\sim 146 \mathrm{~cm}^{-1}$ can

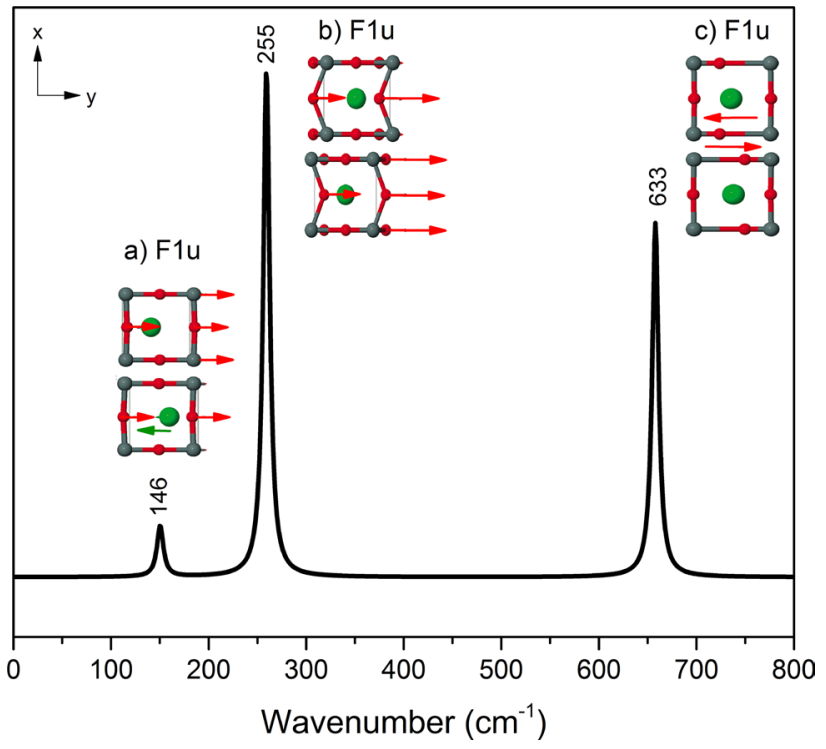

Fig. 4 Active infrared modes. The arrow points in the direction of movement and is relative in scale (no shifts are made)

be assigned to up and down translational movement of $\mathrm{Ba}$ atoms corresponding to the $\mathrm{SnO}_{3}$ (external mode), along with $x-, y$-, and $z$-axes (Fig. 4a). The vibrational mode at $\sim 633 \mathrm{~cm}^{-1}$ corresponds to stretching/compressing vibrations of the $\mathrm{Sn}-\mathrm{O}$ bond along the $x$-, $y$-, and $z$-axes [9, 14, 55-59] (Fig. 4c). The calculated results are consistent with average experimental results [55-57].

\section{Conclusions}

Periodic DFT calculations were carried out using seven different hybrid functionals with a Baranek basis set. Powell's algorithm method was used to perform the optimization of the outermost exponent of the basis set. This methodology can render an improvement in the accuracy of the calculated results of electronic, structural, mechanical, and vibrational properties.

It is expected that this methodology can be applied for further theoretical studies, such as those on surfaces, interfaces, defects, and doping processes in $\mathrm{BaSnO}_{3}$.

The basis set optimization using the PBE0 functional kept the optimized lattice parameter practically unchanged, only needing changes for band gap energy, and produces a considerable improvement in the calculated indirect band gap.

Although there have been no experimental measurements, the elastic constants and $\mathrm{B}_{0}$ are in reasonable agreement with other theoretical results. The calculated IR modes show good agreement with experimental results. 
Acknowledgments This work was supported by Brazilian funding agencies: CNPq, CAPES/PROCAD, and FAPESP (2013/192890, 2013/07296-2). The computational facilities were supported by resources supplied by Molecular Simulations Laboratory, São Paulo State University, Bauru, Brazil.

\section{References}

1. Balamurugan K, Harish Kumar N, Ramachandran B et al (2009) Magnetic and optical properties of Mn-doped $\mathrm{BaSnO}_{3}$. Solid State Commun 149:884-887

2. Cerdà J, Arbiol J, Diaz R et al (2002) Synthesis of perovskitetype $\mathrm{BaSnO}_{3}$ particles obtained by a new simple wet chemical route based on a sol-gel process. Mater Lett 56:131-136

3. Lu W, Jiang S, Zhou D, Gong S (2000) Structural and electrical properties of $\mathrm{Ba}(\mathrm{Sn}, \mathrm{Sb}) \mathrm{O}_{3}$ electroceramics materials. Sens Actuators A Phys 80:35-37

4. Maekawa T, Kurosaki K, Yamanaka S (2006) Thermal and mechanical properties of polycrystalline $\mathrm{BaSnO}_{3}$. J Alloys Compd 416:214-217

5. Zhang WF, Tang J, Ye J (2006) Photoluminescence and photocatalytic properties of $\mathrm{SrSnO}_{3}$ perovskite. Chem Phys Lett 418:174-178

6. Zhang W, Tang J, Ye J (2007) Structural, photocatalytic, and photophysical properties of perovskite $\mathrm{MSnO}_{3}(\mathrm{M}=\mathrm{Ca}, \mathrm{Sr}$, and Ba) photocatalysts. J Mater Res 22:1859-1871

7. Upadhyay S, Parkash O, Kumar Devendra (1997) Preparation and characterization of barium stannate $\mathrm{BaSnO}_{3}$. J Mater Sci Lett 16:1330-1332

8. Smith MG, Goodenough JB, Manthiram A et al (1992) Tin and antimony valence states in $\mathrm{BaSn}_{0.85} \mathrm{Sb}_{0.15} \mathrm{O}_{3-\delta}$. J Solid State Chem 98:181-186

9. Kumari U (2014) Solid-State metathetic synthesis of phase pure $\mathrm{BaSnO}_{3}$ and $\mathrm{BaZrO}_{3}$. Int Res J Pure Appl Chem 3:347-356

10. Huang C, Wang X, Liu X et al (2015) Extensive analysis of the formation mechanism of $\mathrm{BaSnO}_{3}$ by solid-state reaction between $\mathrm{BaCO}_{3}$ and $\mathrm{SnO}_{2}$. J Eur Ceram Soc 36:1-10

11. Udawatte CP, Yoshimura M (2001) Preparation of well-crystallized $\mathrm{BaSnO}_{3}$ powders under hydrothermal conditions. Mater Lett 47:7-10

12. Kutty TRN, Vivekanadan $\mathrm{R}$ (1987) $\mathrm{BaSnO}_{3}$ fine powders from hydrothermal preparations. Mater Res Bull 22:1457-1465

13. Lu W, Schmidt H (2005) Hydrothermal synthesis of nanocrystalline $\mathrm{BaSnO}_{3}$ using a $\mathrm{SnO}_{2} \cdot \mathrm{xH}_{2} \mathrm{O}$ sol. J Eur Ceram Soc 25:919-925

14. Licheron M, Jouan G, Husson E (1997) Characterization of $\mathrm{BaSnO}_{3}$ powder obtained by a modified sol-gel route. J Eur Ceram Soc 17:1453-1457

15. Wang W, Liang S, Ding K et al (2014) Microwave hydrothermal synthesis of $\mathrm{MSnO}_{3}\left(\mathrm{M}^{2+}=\mathrm{Ca}^{2+}, \mathrm{Sr}^{2+}, \mathrm{Ba}^{2+}\right)$ : effect of $\mathrm{M}^{2+}$ on crystal structure and photocatalytic properties. J Mater Sci 49:1893-1902

16. Lim CS (2013) A solvothermal process to synthesize barium metastannate nanoparticles assisted by microwave irradiation. Asian J Chem 25:2363-2365

17. Mizoguchi H, Eng HW, Woodward PM (2004) Probing the electronic structures of ternary perovskite and pyrochlore oxides containing $\mathrm{Sn}^{4+}$ or $\mathrm{Sb}^{5+}$. Inorg Chem 43:1667-1680

18. Liu Q, Li B, Liu J et al (2012) Structure and band gap tuning of transparent $\left(\mathrm{Ba}_{1-\mathrm{x}} \mathrm{Sr}_{\mathrm{x}}\right) \mathrm{SnO}_{3}$ thin films epitaxially grown on $\mathrm{MgO}$ substrates. Europhys Lett Assoc 98:47010-47014

19. Liu Q, Dai J, Liu Z et al (2010) Electrical and optical properties of $\mathrm{Sb}$-doped $\mathrm{BaSnO}_{3}$ epitaxial films grown by pulsed laser deposition. J Phys D Appl Phys 43:455401-455408
20. Prodjosantoso AK, Zhou Q, Kennedy BJ (2013) Synchrotron $\mathrm{X}$-ray diffraction study of the $\mathrm{Ba}_{1-\mathrm{x}} \mathrm{SrSnO}_{3}$ solid solution. J Solid State Chem 200:241-245

21. Smith AJ, Welch AJE (1960) Some mixed metal oxides of perovskite structure. Acta Crystallogr 13:653-656

22. Moreira E, Henriques JM, Azevedo DL et al (2012) Structural and electronic properties of $\mathrm{Sr}_{\mathrm{x}} \mathrm{Ba}_{1-\mathrm{x}} \mathrm{SnO}_{3}$ from first principles calculations. J Solid State Chem 187:186-194

23. Bouhemadou A, Haddadi K (2010) Structural, elastic, electronic and thermal properties of the cubic perovskite-type $\mathrm{BaSnO}_{3}$. Solid State Sci 12:630-636

24. Wang YZ, Bevillon E, Chesnaud A et al (2009) Atomistic simulation of pure and doped $\mathrm{BaSnO}_{3}$. J Phys Chem C 113:20486-20492

25. Soleimanpour S, Kanjouri F (2014) First principle study of electronic and optical properties of the cubic perovskite $\mathrm{BaSnO}_{3}$. Phys B Condens Matter 432:16-20

26. Singh DJ, Xu Q, Ong KP (2014) Strain effects on the band gap and optical properties of perovskite $\mathrm{SrSnO}_{3}$ and $\mathrm{BaSnO}_{3}$. Appl Phys Lett 104:1-5

27. Singh DJ, Papaconstantopoulos DA, Julien JP, Cyrot-Lackmann $\mathrm{F}$ (1991) Electronic structure of $\mathrm{Ba}(\mathrm{Sn}, \mathrm{Sb}) \mathrm{O}_{3}$ Absence of superconductivity. Phys Rev B 44:9519-9523

28. Kim BG, Jo JY, Cheong SW (2013) Hybrid functional calculation of electronic and phonon structure of $\mathrm{BaSnO}_{3}$. J Solid State Chem 197:134-138

29. Maul J, Santos IMG, Sambrano JR, Erba A (2016) Thermal properties of the orthorhombic $\mathrm{CaSnO}_{3}$ perovskite under pressure from $a b$ initio quasi-harmonic calculations. Theor Chem Acc 135:36-45

30. Maul J, Erba A, Santos IMG et al (2015) In silico infrared and Raman spectroscopy under pressure: the case of $\mathrm{CaSnO}_{3}$ perovskite. J Chem Phys 142:014505-014514

31. Heifets E, Eglitis RI, Kotomin EA et al (2002) First-principles calculations for $\mathrm{SrTiO}_{3}\left(\begin{array}{lll}1 & 0 & 0\end{array}\right)$ surface structure. Surf Sci 513:211-220

32. Heifets E, Goddard WA, Kotomin EA et al (2004) Ab initio calculations of the $\mathrm{SrTiO}_{3}\left(\begin{array}{lll}1 & 1 & 0\end{array}\right)$ polar surface. Phys Rev B 69:235417-235425

33. Becke AD (1993) Density-functional thermochemistry. III. The role of exact exchange. J Chem Phys 98:5648-5652

34. Lee C, Yang W, Parr RG (1988) Development of the Colle-Salvetti correlation-energy formula into a functional of the electron density. Phys Rev B 37:785-789

35. Bilc DI, Orlando R, Shaltaf R et al (2008) Hybrid exchangecorrelation functional for accurate prediction of the electronic and structural properties of ferroelectric oxides. Phys Rev B 77:165107-165120

36. Perdew JP (1991) Unified theory of exchange and correlation beyond the local density approximation. In: Ziesche P, Eschrig $\mathrm{H}$ (eds) Electronic structure solids'91, vol 17. Akademie, Berlin, pp 11-20

37. Perdew JP, Yue W (1989) Accurate and simple density functional for the electronic exchange energy: generalized gradient approximation. Phys Rev B 40:3399

38. Perdew JP, Wang Y (1992) Accurate and simple analytic representation of the electron-gas correlation energy. Phys Rev B 45:13244-13249

39. Adamo C, Barone V (1999) Toward reliable density functional methods without adjustable parameters: the PBE0 model. J Chem Phys 110:6158-6170

40. Zhao Y, Truhlar DG (2008) Construction of a generalized gradient approximation by restoring the density-gradient expansion and enforcing a tight Lieb-Oxford bound. J Chem Phys 128:184109-184117

41. Demichelis R, Civalleri B, Ferrabone M, Dovesi R (2010) On the performance of eleven DFT functionals in the description of the 
vibrational properties of aluminosilicates. Int J Quantum Chem 110:406-415

42. Dovesi R, Orlando R, Erba A et al (2014) CRYSTAL14: a program for the ab initio investigation of crystalline solids. Int $\mathbf{J}$ Quantum Chem 114:1287-1317

43. Towler MD, Zupan A, Causà M (1996) Density functional theory in periodic systems using local Gaussian basis sets. Comput Phys Commun 98:181-205

44. Sophia G, Baranek P, Sarrazin C et al (2013) First-principles study of the mechanisms of the pressure-induced dielectric anomalies in ferroelectric perovskites. Phase Transit 86:1069-1084

45. Sophia G (2014) Ab-initio study of the dielectric response of high-permittivity perovskites for energy storage. Università degli Studi di Torino, Turin

46. Huang W, Yang H, Lu G, Gao Y (2013) Theoretical calculations of the linear, nonlinear elastic constants and the Grüneisen parameters of cubic perovskites $(\mathrm{Sr}, \mathrm{Ba}, \mathrm{Pb}) \mathrm{TiO}_{3}$. Phys B Condens Matter 411:56-61

47. Bévillon É, Chesnaud A, Wang Y et al (2008) Theoretical and experimental study of the structural, dynamical and dielectric properties of perovskite $\mathrm{BaSnO}_{3}$. J Phys: Condens Matter 20:145217-145223

48. Sambrano J, Toniatto E (2014) First-principles simulation of elastic constants and electronic properties of GaN. Curr Phys Chem 4:65-70

49. Marana NL, Sambrano JR, de Souza AR (2010) Propriedades eletrônicas, estruturais e constantes elásticas do $\mathrm{ZnO}$. Quim Nova 33:810-815
50. Murnaghan FD (1944) The compressibility of media under extreme pressures. Proc Natl Acad Sci 30:244-247

51. Birch F (1947) Finite elastic strain of cubic crystals. Phys Rev 71:809-824

52. Birch F (1978) Finite strain isotherm and velocities for singlecrystal and polycrystalline $\mathrm{NaCl}$ at high pressures and $300 \mathrm{~K}$. J Geophys Res 83:1257-1268

53. Poirier J-P, Tarantola A (1998) A logarithmic equation of state. Phys Earth Planet Inter 109:1-8

54. Vinet P, Ferrante J, Smith JR, Rose JH (1986) A universal equation of state for solids. J Phys C: Solid State Phys 29:2963-2969

55. Deepa AS, Vidya S, Manu PC et al (2011) Structural and optical characterization of $\mathrm{BaSnO}_{3}$ nanopowder synthesized through a novel combustion technique. J Alloys Compd 509:1830-1835

56. Marikutsa A, Rumyantseva M, Baranchikov A, Gaskov A (2015) Nanocrystalline $\mathrm{BaSnO}_{3}$ as an alternative gas sensor material: surface reactivity and high sensitivity to $\mathrm{SO}_{2}$. Materials (Basel) 8:6437-6454

57. Lu WS, Schmidt H (2007) Preparation and characterization of $\mathrm{BaSnO}_{3}$ powders by hydrothermal synthesis from tin oxide hydrate gel. J Mater Sci 42:10007-10013

58. Ansaree MJ, Upadhyay S (2015) Electrical characterization of porous La-doped $\mathrm{BaSnO}_{3}$ using impedance spectroscopy. Ionics (Kiel) 21:2825-2838

59. Ochoa Muñoz YH, Ponce M, Rodríguez Páez JE (2015) Comparative study of two wet chemical methods of $\mathrm{BaSnO}_{3}$ synthesis: mechanism of formation of mixed oxide. Powder Technol 279:86-95 